\title{
Study in the sodium hyaluronate attenuating the functional recovery and thermal hyperalgesia by alterations the inflammation and collagen fiber in the osteoarthritis rats
}

\author{
Jin-Hee Han, In-Duk Oh, Myung-Soo Jang, Jun-Young Chung, Jae-Woo Yi \\ Department of Anesthesiology and Pain Medicine, Kyung Hee Medical Center, \\ Kyung Hee University College of Medicine, Seoul, Republic of Korea
}

\section{Introduction}

Osteoarthritis (OA) is a complex disease of the entire joint, affecting the cartilage, bone and synovial membranes. Inflammation of articular cavity is accompanied to pathological changes that have a critical impact on neuropathic pain and locomotion disorders. The sodium hyaluronate (SH) is similar to a substance that occurs naturally in the joint. It may work by acting as a lubricant and shock absorber in the joint, helping the knee to move smoothly, thereby lessening pain. However, current studies have limited information about mechanisms of this SH. Therefore, we investigated the effects of sodium hyaluronate on locomotor function, hyperalgesia degree, and expressions of inflammation and collagen fibers (type I and II) in the articular cavity following osteoarthritis rats.

\section{Material \& Methods}

Animals \& grouping

Male Sprague-Dawley rats (10 weeks, $250 \pm 10 \mathrm{~g}$ ) $\mathbf{N}=\mathbf{5 0}$ ( $\mathrm{n}=10$ in each group)

\begin{tabular}{|l|l|l|l|l|} 
CON & OA & OA-10mg/ml SH & OA-15mg/ml SH & OA-20mg/ml SH \\
\hline
\end{tabular}

Induction of osteoarthritis

OA induced rats received an intraarticular injection of monosodium iodoacetate (MIA; $3 \mathrm{mg} / \mathrm{kg}$, volume: $60 \mathrm{ul}$ ) in right knee joint.

\section{Experimental schedule}

\section{$\because=$ Weight load test / Walking track analysis}

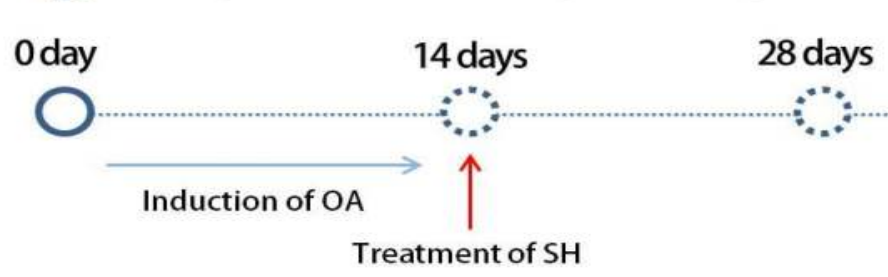

Functional tests

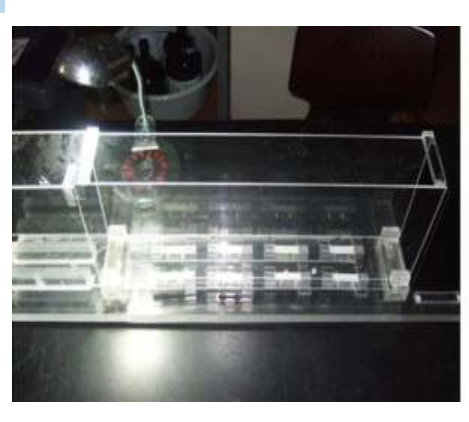

Fig. 2. Weight load test

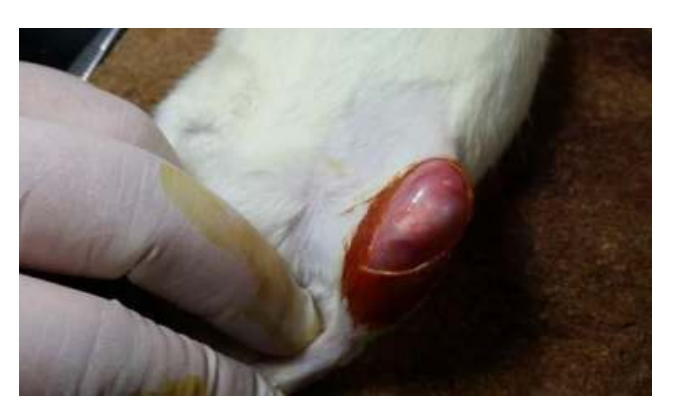

Fig. 1. Induction of $O A$
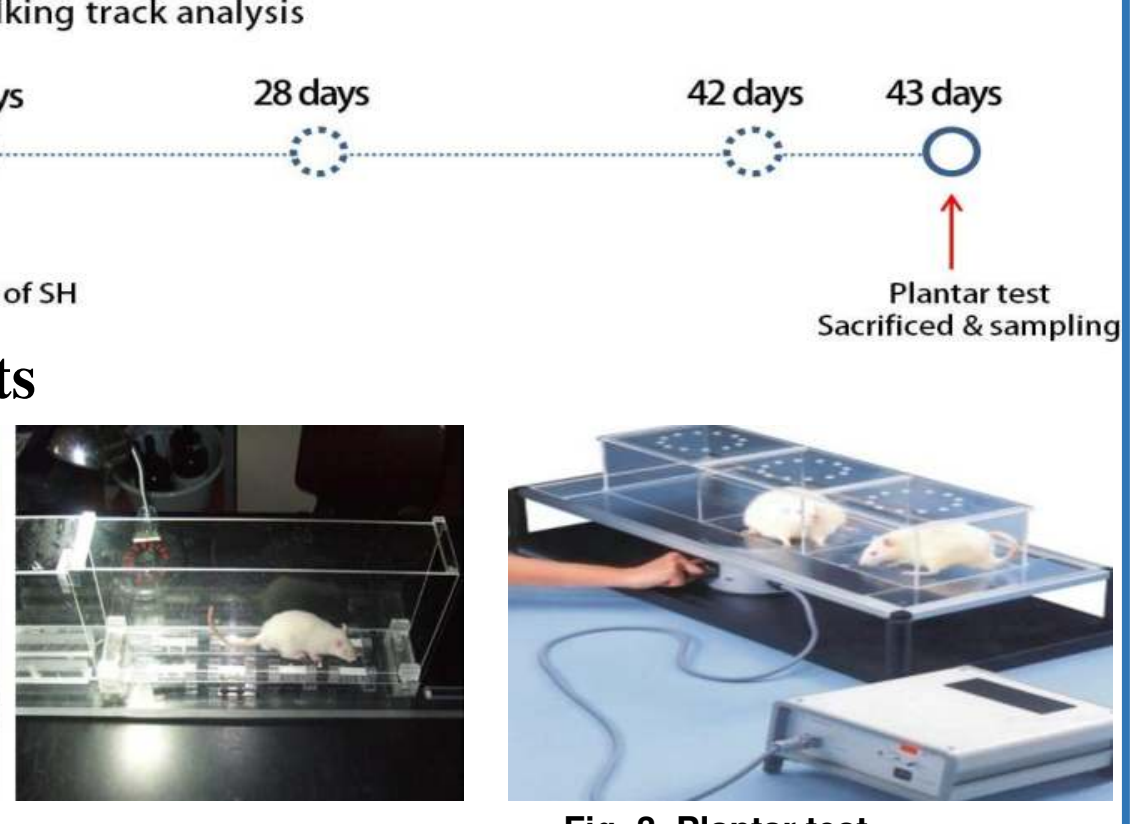

Fig. 3. Plantar test

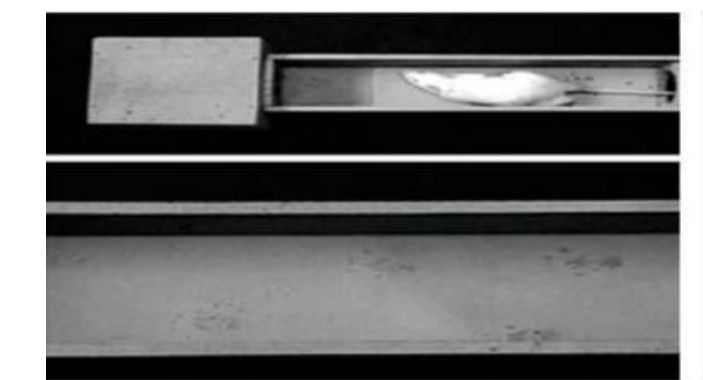

Fig. 4. Walking track analysis

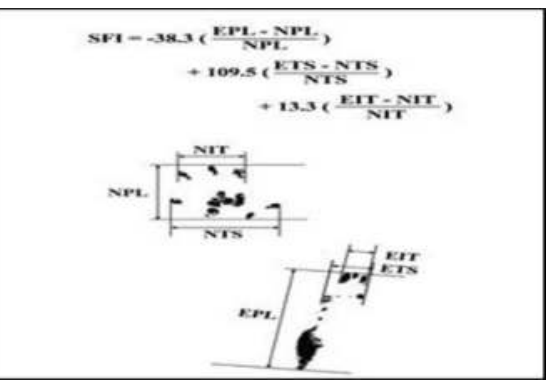

Data analysis

Hematoxylin \& eosin (H\&E) staining

Masson's trichrome (MT) staining

Immunohistochemistry of collagen fiber I and II

SPSS (ver. 21.0), One-way ANOVA, Duncan's Post-hoc

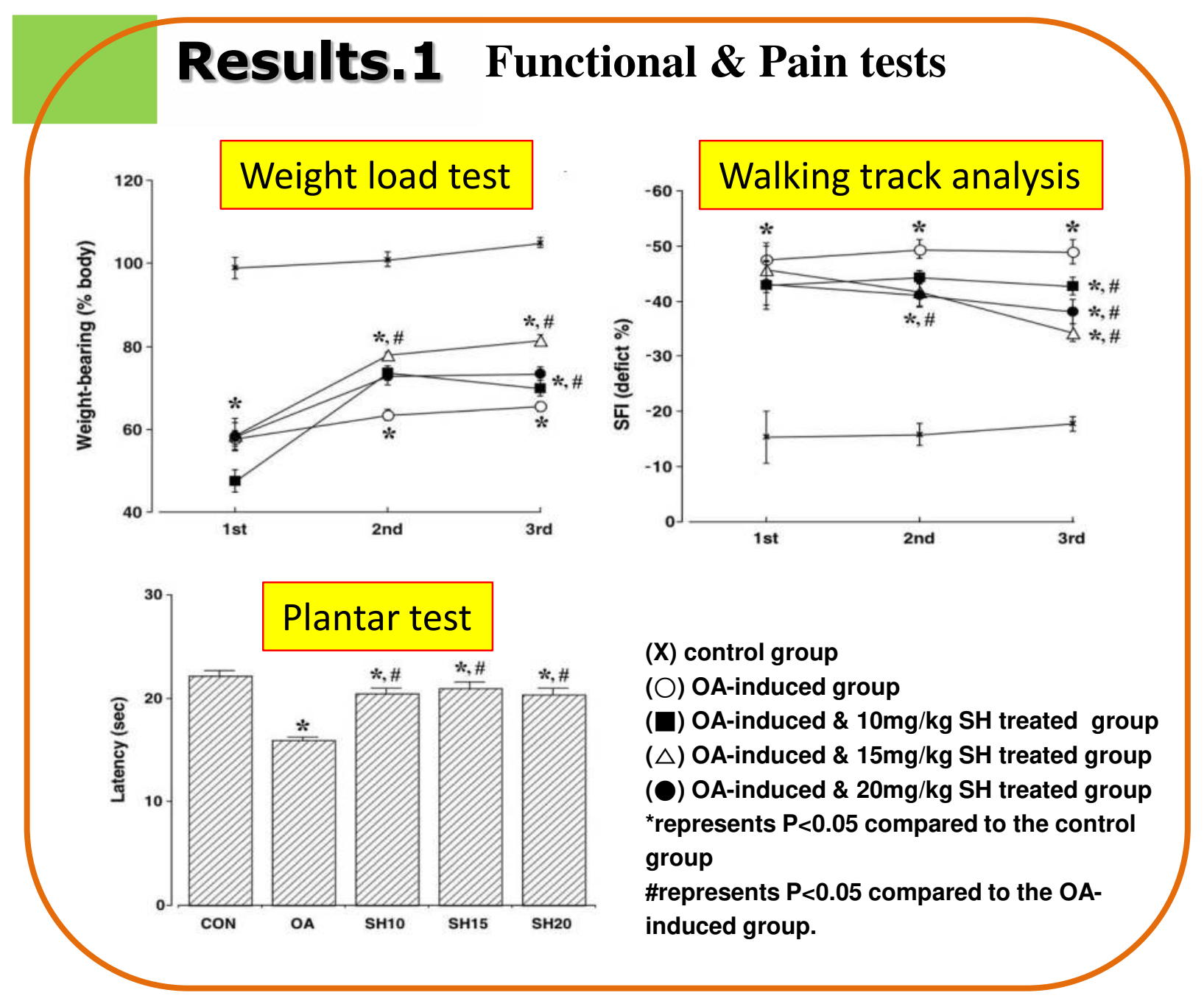

Results. 2 Histology \& Collagen fiber I,II

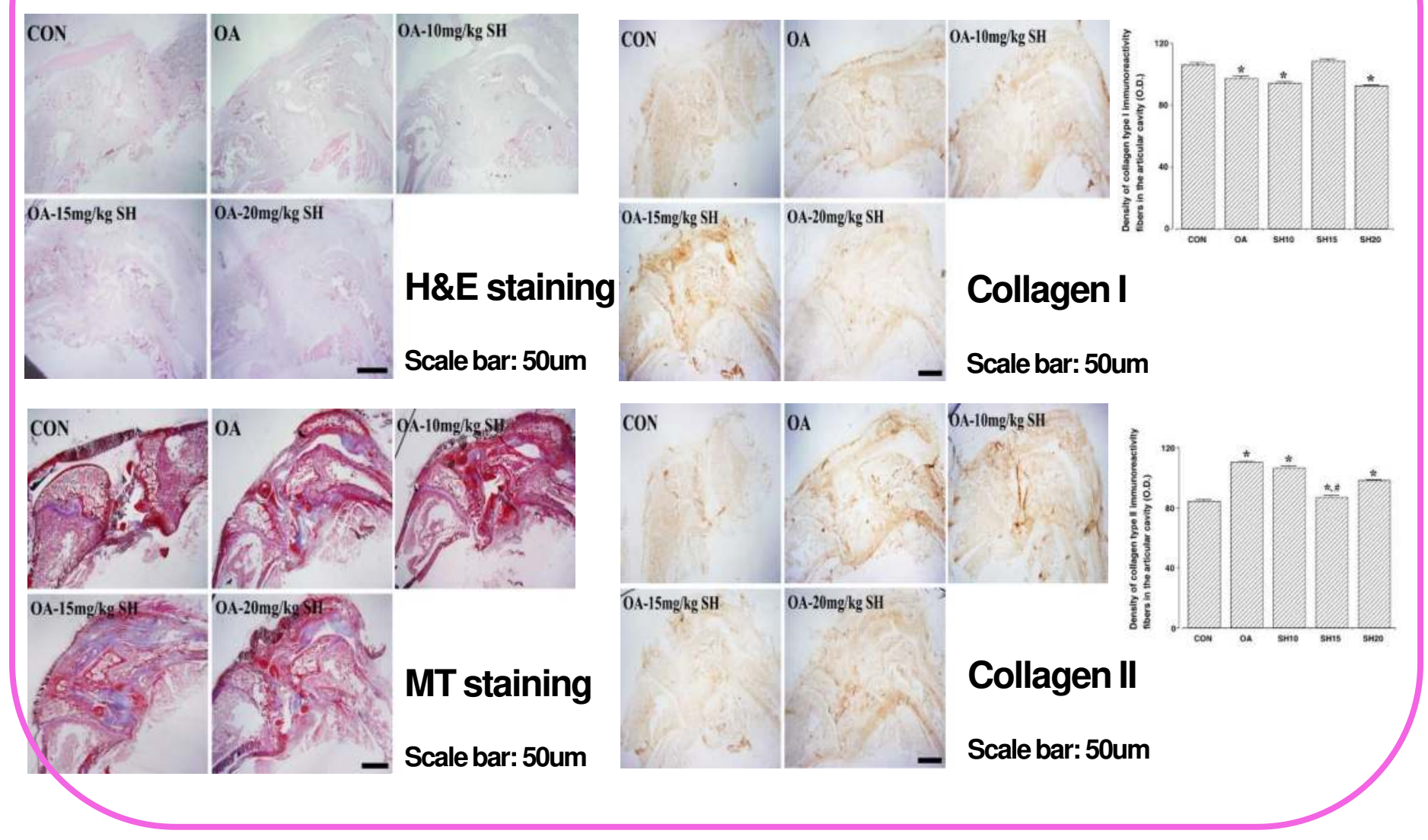

\section{Conclusion}

These results showed that SH facilitates functional recovery and inhibits pain susceptibility by suppressing inflammation, and modulating collagen fibers in $\mathrm{OA}$. We suggest that $\mathrm{SH}$ might be considered as a new therapeutic intervention for functional recovery and pain control in $\mathrm{OA}$. 